\title{
Ciencia y tecnología del agua desde la práctica
}
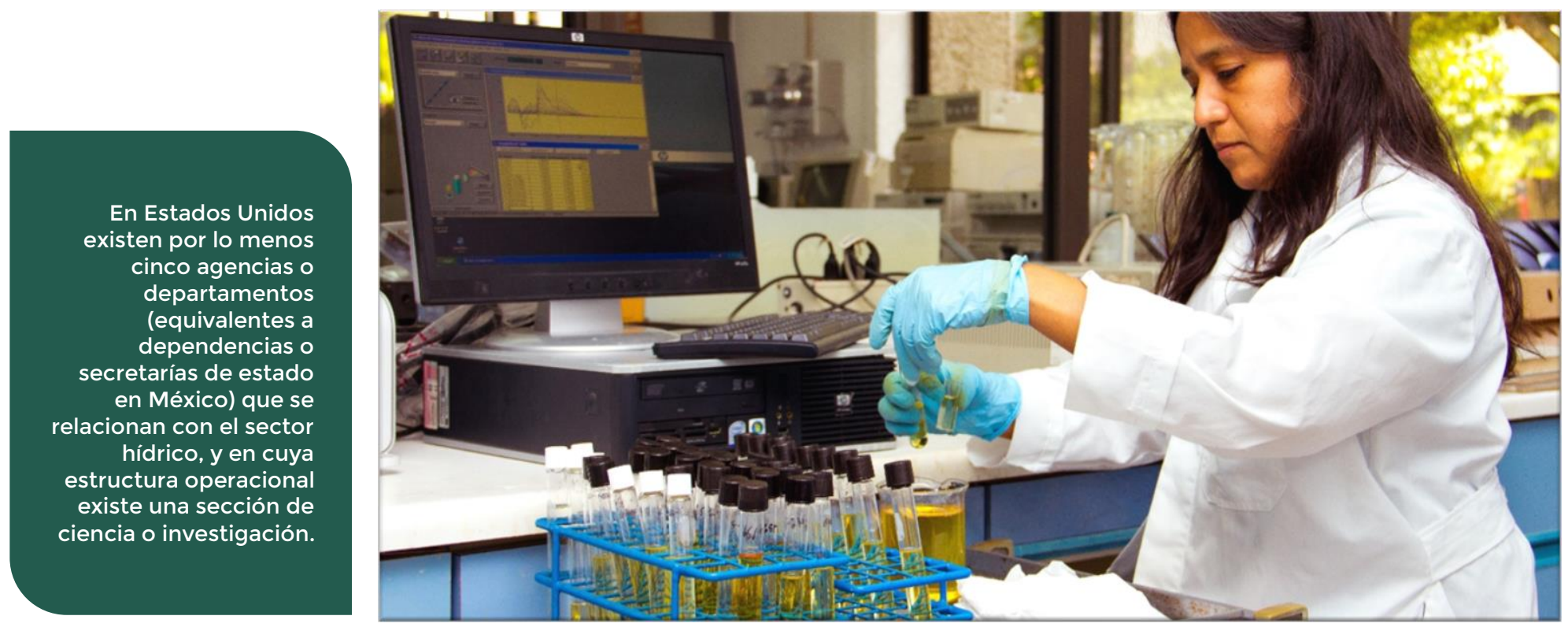

Las disciplinas científicas asociadas al sector hídrico desempeñan un papel primordial en la mitigación o solución de problemas de sostenibilidad ambiental.

No obstante, para ver un impacto real de la ciencia y tecnología en la solución de problemas es necesario contar con mecanismos efectivos de acompañamiento y comunicación de la ciencia. Los grandes problemas hídricos que aquejan a México, tales como la escasez permanente de agua en una buena parte del territorio nacional (sin mencionar las implicaciones negativas en términos de gobernanza y conflictividad social), los problemas de salud pública asociados a la mala calidad en fuentes y redes de abastecimiento, así como la poca adaptación al cambio climático que actualmente prevalece, entre otros, requieren de una mayor interacción entre la evidencia (basada en la ciencia y tecnología) y la toma de decisiones (a través de políticas públicas) (von Schneidemesser et al., 2020).

Es importante mencionar que la ciencia y sus instituciones pueden llegar a entrar en crisis, y en esos casos es necesario reformar el sistema de ciencia e investigación prevaleciente previo a dicha crisis. Tal es el caso de Japón, en dónde la confianza pública y política hacia sus universidades y centros de investigación tuvo un colapso como consecuencia del terremoto, tsunami y desastre nuclear ocurridos en marzo de 2011. La falta de preparación ante este "cisne negro", provocado por una cadena de eventos 
"posibles, pero no probables", de acuerdo con las autoridades y sus asesores científicos, puso en entredicho la utilidad de la ciencia y la tecnología al servicio de la población de este país (Arimoto y Sato, 2014). En los años posteriores a este desastre, el gobierno japonés llevó a cabo una reforma en el sector de la ciencia e investigación (uno de los más importantes del mundo) con el objetivo de reconstruir la confianza entre ciencia y sociedad.

No obstante, la coordinación y comunicación entre dos ecosistemas completamente diferentes: el científico (enfocado en desarrollar investigación básica y aplicada o realizar evaluaciones científicas, entre otros) y el de gestión y regulación (encargados de trabajar con otras dependencias gubernamentales, apoyar en la redacción e implementación de nuevas leyes, entre otros) es posible mediante la creación de instituciones en donde la ciencia, la tecnología y la innovación ocupen un lugar preponderante dentro de dichas organizaciones.

Por ejemplo, en Estados Unidos existen por lo menos cinco agencias o departamentos (equivalentes a dependencias o secretarías de estado en México) que se relacionan con el sector hídrico, y en cuya estructura operacional existe una sección de ciencia o investigación. A continuación, se describirá de manera breve las funciones contempladas en cada sección:

1. Oficina de Investigación y Desarrollo (ORD, por sus siglas en inglés), perteneciente a la Agencia de Protección Ambiental (Environmental Protection Agency, EPA): Esta oficina lleva a cabo investigación para la EPA con el objetivo de proveer con credibilidad la base para la toma de decisiones asociadas a las salvaguardas de la salud humana y ecosistemas con respecto a los contaminantes ambientales. La oficina está compuesta por seis áreas de investigación, dentro de las cuales una se enfoca únicamente al tema de la sostenibilidad de los recursos hídricos.

2. 2. Área de Misión de Recursos Hídricos (WRMA, por sus siglas en inglés) perteneciente al Servicio Geológico de EU (US Geological Survey, USCS): Esta área proporciona la información científica requerida para el bienestar económico local y nacional, la protección de la vida y la infraestructura, y la gestión efectiva de los recursos hídricos de los EU. A través de asociaciones con socios regionales se realizan acciones de monitoreo, evaluación, investigación específica y divulgación de información sobre diferentes temáticas, tales como las aguas superficiales y subterráneas, así como la disponibilidad hídrica y su uso consuntivo.

3. Oficina del Científico en Jefe (OCS, por sus siglas en inglés), perteneciente a la Administración Nacional de Aeronáutica y el Espacio (NASA): El científico en jefe sirve como el principal asesor del titular de la Administración en programas de ciencia e investigación impulsados por esta agencia y en planeación estratégica, entre otros. También representa los objetivos y logros ante la comunidad nacional e internacional, incluyendo otras agencias gubernamentales y organizaciones científicas, así como a los sectores público, privado y académico.

4. Investigación Oceánica y Atmosférica (OAR, por sus siglas en inglés), perteneciente a la Oficina Nacional de Administración Oceánica y Atmosférica (NOAA): La OAR promueve el mejoramiento de las predicciones climatológicas y oceánicas, las alertas tempranas de desastres naturales, así como una mejor comprehensión de nuestro sistema terrestre 
mediante el uso de la mejor ciencia disponible y libre de sesgos. Entre sus objetivos específicos se encuentran el monitoreo de la variabilidad y cambio climático para fines de planeación y respuesta, la gestión, protección y restauración de los recursos costeros y oceánicos mediante soluciones basadas en la naturaleza, así como el apoyo al sector del transporte terrestre y marítimo mediante información confiable y segura.

5. Oficina del Científico en Jefe (OCS, por sus siglas en inglés), perteneciente al Departamento de Agricultura de los Estados Unidos (USDA): El científico en jefe informa al titular del USDA acerca de los impactos al sistema agropecuario de los Estados Unidos, sus comunidades y ecosistemas aledaños, derivados de las decisiones, políticas y regulaciones que el gobierno federal lleva a cabo. Esta oficina cuenta con la autoridad científica, que asegura que la asesoría y consultoría entregada al Departamento de Agricultura y a sus usuarios se apega a los estándares más altos de rigor intelectual e integridad científica.

Todos los ejemplos que se acaban de presentar previamente indican que las organizaciones gubernamentales necesitan la mayor cantidad de evidencia científica para todo tipo de decisiones, principalmente aquellas orientadas a brindar el mayor beneficio a nuestra sociedad (Rosen, 2018). Tal como se muestra en la siguiente figura, existe un amplio espectro de posibilidades para involucrarse de manera conjunta en el mejoramiento de la toma de decisiones y de políticas públicas, mediante un diálogo permanente con representantes del poder ejecutivo y legislativo, respectivamente. 


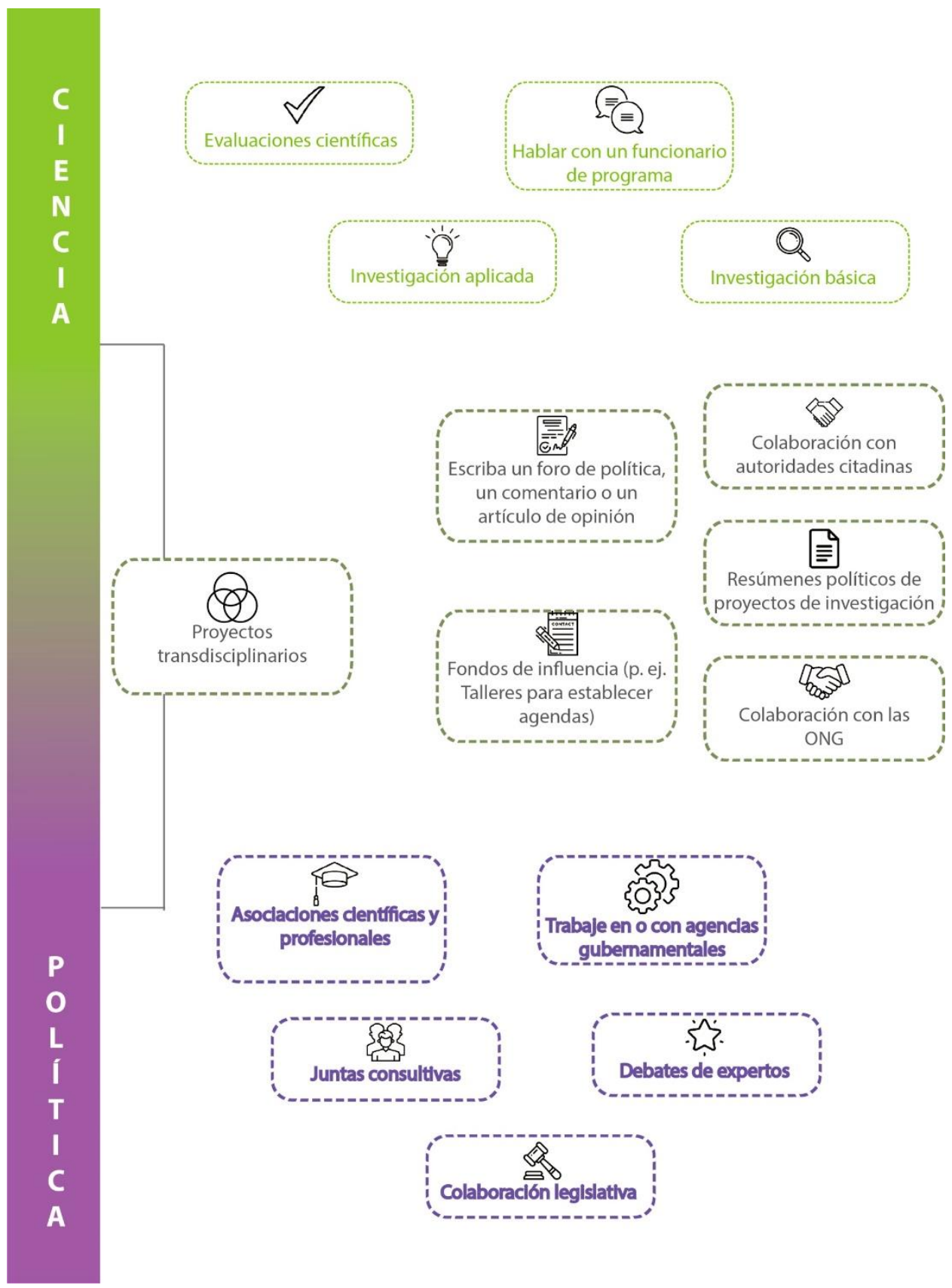

Fuente: von Schneidemesser et al., 2020

En el caso del sector ambiental, es necesario estrechar las relaciones entre los organismos científicotecnológicos y las instituciones encargadas de administrar, regular, controlar y proteger los recursos naturales. En este sentido, es de suma importancia repensar los retos y oportunidades que implican hacer ciencia desde la práctica, esto es, transformar la evidencia científico-tecnológica en decisiones de alto impacto y para el beneficio de todos, de la mano de los formuladores de políticas y tomadores de decisiones. 
Referencias

Arimoto, T. y Sato, Y. (2014). Crisis, renewal and the prospects for science advice in Japan. The Guardian. https://www.theguardian.com/science/political-science/2014/aug/29/crisis-renewal-and-theprospects-for-science-advice-in-japan

Rosen, J. (2018). Help to shape policy with your science. Nature, 560(7720), 671-673. Career Feature. https://doi.org/10.1038/d41586-018-06038-4

von Schneidemesser, E., Melamed, M., y Schmale, J. (2020). Prepare Scientists to Engage in Science-Policy. Earth's Future, 8, e2020EF001628. https://doi.org/10.1029/2020EF001628 